\title{
Time-adaptive high-order compact finite difference schemes for option pricing in a family of stochastic volatility models
}

\author{
Bertram Düring and Christof Heuer
}

\begin{abstract}
We propose a time-adaptive high-order compact finite difference scheme for option pricing in a family of stochastic volatility models. We employ a semidiscrete high-order compact finite difference method for the spatial discretisation, and combine this with an adaptive time discretisation, extending ideas from [LSRHF02] to fourth-order multistep methods in time.
\end{abstract}

\section{Introduction}

Stochastic volatility models have become one of the standard approaches for financial option pricing. They are based on a two-dimensional stochastic diffusion process containing two Brownian motions with correlation $\rho \in[-1,1]$, i.e. $\mathrm{E}\left[\mathrm{d} W_{1}(t) \mathrm{d} W_{2}(t)\right]=\rho \mathrm{d} t$, on a given filtered probability space for the underlying asset $S=S(t)$ and the stochastic variance $v=v(t)$. In this chapter we consider the following class of stochastic volatility models,

$$
d S=\mu S \mathrm{~d} t+\sqrt{v} S \mathrm{~d} W_{1}, \quad d v=\kappa v^{a}(\theta-v) \mathrm{d} t+\sigma v^{b} \mathrm{~d} W_{2},
$$

with given drift $\mu \in \mathbb{R}$ of the underlying $S(t)$, long run mean $\theta>0$, mean reversion speed $\kappa>0$, and volatility of volatility $\sigma>0$, see e.g. [CJM10]. Additionally, it holds $a \geq 0$ and $b \in(0,3 / 2]$. Many well-known models are included in the family (1). The prominent Heston (or $S Q R$ ) model [Hes93] is obtained for $a=0, b=1 / 2$. Other known models include the GARCH (or VAR) model [Dua95], with $a=0$, $b=1$, and the 3/2-model [Lew00] in which $a=0, b=3 / 2$. There are also models

Bertram Düring

Mathematics Institute, University of Warwick, Coventry, CV4 7AL, United Kingdom, e-mail: bertram.during@warwick.ac.uk

Christof Heuer

Frankfurt, Germany, e-mail: heuer.chr@googlemail.com 
with non-linear mean reversion, following [CJM10], we denote these models as the SQR-N model $(a=1, b=1 / 2), \operatorname{VAR}-N$ model $(a=1, b=1)$, and $3 / 2-N$ model $(a=1, b=3 / 2)$.

For the family of stochastic volatility models (1), application of Itô's Lemma and standard arbitrage arguments lead to partial differential equations for the option price $V=V(S, v, t)$, which are of the following form

$\frac{\partial V}{\partial t}+\frac{v S^{2}}{2} \frac{\partial^{2} V}{\partial S^{2}}+\rho \sigma v^{b+\frac{1}{2}} S \frac{\partial^{2} V}{\partial S \partial v}+\frac{\sigma^{2} v^{2 b}}{2} \frac{\partial^{2} V}{\partial v^{2}}+r S \frac{\partial V}{\partial S}+\kappa v^{a}(\theta-v) \frac{\partial V}{\partial v}-r V=0$,

where $r \geq 0$ denotes the risk-free interest rate. Equation (2) has to be solved (backward in time) for $S, v>0,0 \leq t<T$, with an expiration date $T>0$, and subject to final and boundary conditions depending on the specific option considered. In the case of a European Put options, for example, the final condition is given by $V(S, v, T)=\max (K-S, 0)$ with strike price $K>0$.

In the mathematical literature, there are many works on numerical methods for option pricing in one-dimension (single risk factor), but less papers considering numerical methods for option pricing in stochastic volatility models, i.e. for two spatial dimensions. Finite difference approaches used are often standard, low order methods, i.e. second order in space. In the last decade, high-order (fourth order in space) compact finite difference discretisations for option pricing in stochastic volatility models have been presented, e.g. in [DF12, DH15]. We refer to [DH15] for an overview of the finite difference literature and other methods.

The originality of the present chapter consists in proposing a new, time-adaptive high-order compact finite difference scheme for option pricing in a family of stochastic volatility models. Our approach builds on ideas from [DH15] and [LSRHF02]. We employ a semi-discrete high-order compact finite difference method for the spatial discretisation, using the methodology developed in [DH15]. For the adaptive time discretisation, we follow basic ideas of [LSRHF02], where two-step methods for the time-discretisation were used, and generalise this approach to consider fourth-order multistep methods in time. We obtain a time-adaptive high-order compact scheme that is fourth order accurate in both space and time.

\section{Transformation of the partial differential equation}

We first transform $\tau=T-t$, and $u=\exp (r \tau) V / K$ in (2). Depending on the model parameter $b$, we apply subsequent transformations, in such a way that the second derivatives in $x$ - and $y$-direction share the same coefficient.

For $b \neq 3 / 2$ we apply the transformations $x=(3 / 2-b) \ln (S / K), y=v^{3 / 2-b} / \sigma$, and arrive at

$$
u_{\tau}+a(y)\left(u_{x x}+u_{y y}\right)+b(y) u_{x y}+c_{1}(y) u_{x}+c_{2}(y) u_{y}=0,
$$

to be solved on the rectangular spatial domain $\Omega=\left(x_{\min }, x_{\max }\right) \times\left(y_{\min }, y_{\max }\right)$, with 


$$
\begin{aligned}
a(y)= & -\sigma^{\frac{-5+2 b}{-3+2 b}} y^{-2(-3+2 b)^{-1}}(-3+2 b)^{2}(8 \sigma)^{-1}, \quad b(y)=2 \rho a(y), \\
c_{1}(y)= & (3-2 b)\left(\sigma^{-\frac{-5+2 b}{-3+2 b}} y^{-2(-3+2 b)^{-1}}-2 r \sigma\right)(4 \sigma)^{-1}, \\
c_{2}(y)= & (3-2 b)\left(2 \sigma^{\frac{-5+2 b}{-3+2 b}} y^{-\frac{-1+2 b}{-3+2 b}} b-4 \sigma^{-\frac{1+2 a-2 b}{-3+2 b}} y^{-\frac{1+2 a-2 b}{-3+2 b}} \kappa \theta\right. \\
& \left.+4 \sigma^{-\frac{3+2 a-2 b}{-3+2 b}} y^{-\frac{3+2 a-2 b}{-3+2 b}} \kappa-\sigma^{\frac{-5+2 b}{-3+2 b}} y^{-\frac{-1+2 b}{-3+2 b}}\right)(8 \sigma)^{-1},
\end{aligned}
$$

and subject to $u(x, y, 0)=\max (1-\exp (x /(3 / 2-b)), 0)$.

For $b=3 / 2$, we apply the transformations $x=\ln (S / K), y=\ln (v) / \sigma$, and obtain (3) with coefficients $a(y)=-\exp (\sigma y) / 2, b(y)=-\rho \exp (\sigma y), c_{1}(y)=\exp (\sigma y) / 2-$ $r, c_{2}(y)=\left(\sigma^{2} \exp (\sigma y)-2 \kappa \theta \exp (\sigma y(a-1))+2 \kappa \exp (a \sigma y)\right) /(2 \sigma)$ and subject to $u(x, y, 0)=\max \left(1-e^{x}, 0\right)$.

\section{Time-adaptive high-order compact scheme}

We use the high-order compact semi-discrete (discretising in space only) scheme from [DH15] for (3). Since the coefficients of $u_{x x}$ and $u_{y y}$ in (3) are identical, results from [DH15] show that the scheme provides a fourth-order accurate spatial discretisation employing a uniform grid with $h_{1}=h_{2}=h>0$. The semi-discrete scheme can be written in matrix form as

$$
M_{h} \partial_{\tau} U_{h}(\tau)=g^{(h)}(\tau)-K_{h} U^{(h)}(\tau)=: F(\tau)
$$

The known vector $g^{(h)}$ has only non-zero entries due to the influence of the boundary conditions and the matrices $M_{h}$ and $K_{h}$ do not depend on $\tau$.

At the boundary $x=x_{\min }$ and $x=x_{\max }$ we impose Dirichlet type boundary conditions. For $y=y_{\min }$ or $y=y_{\max }$ we do not impose any boundary condition, but apply the discretisation of the spatial interior. The resulting ghost points are extrapolated from the interior with sufficiently high order. Due to the low regularity of the typical initial conditions, we employ a smoothing operator [KTW70] to ensure fourth-order spatial convergence. For further details of the implementation of boundary and initial conditions, we refer to [DH15].

Our approach for time adaptivity is motivated by [LSRHF02], where two-step methods are used for time discretisation. Here, to match the fourth-order accuracy in space, we consider fourth-order multistep methods in time. We approximate the system of ordinary differential equations (4) using fourth-order multistep methods and variable, adaptive time step sizes. In each time step, we use a (numerically cheap) predictor scheme to estimate the local truncation error, adapt the time step accordingly, and then solve using a corrector scheme. Necessary start-up values are computed using a Crank-Nicolson time-discretisation.

Predictor scheme. Consider $\tau_{\min }=\tau_{0}<\tau_{1}<\ldots<\tau_{j}$ with $j \geq 4$ and $\tau_{j}<\tau_{\max }$ in time with the step sizes $k_{n}=\tau_{n}-\tau_{n-1}>0$ for $n=1, \ldots, j$. We denote the value of the vector $U^{(h)}$ at time $\tau_{n}$ by $U_{n}^{(h)}$. 
We use a four-step predictor scheme with (non-equidistant) time steps,

$$
\alpha_{0}^{(\mathrm{pre})} M_{h} U_{n}^{(h)}=k_{n} g_{n-1}^{(h)}-\left[\alpha_{1}^{(\mathrm{pre})} M_{h}+k_{n} K_{h}\right] U_{n-1}^{(h)}-M_{h} \sum_{j=2}^{4} \alpha_{j}^{(\mathrm{pre})} U_{n-j}^{(h)}
$$

where

$$
\begin{aligned}
& \alpha_{0}^{(\mathrm{pre})}=2 \imath_{1} \imath_{2} \iota_{3}+\imath_{3} \imath_{1}^{2}+l_{2} \imath_{1}^{2}+\imath_{2}^{2} \iota_{3}+\imath_{1} \imath_{2}^{2} / \varphi_{0}^{(\mathrm{pre})},
\end{aligned}
$$

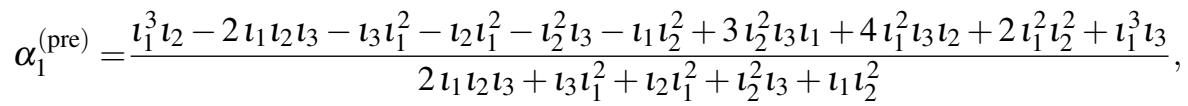

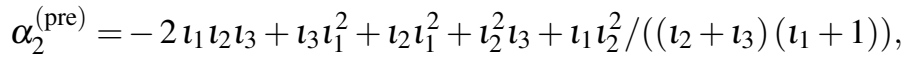

$$
\begin{aligned}
& \alpha_{3}^{(\mathrm{pre})}=l_{2}^{2}\left(l_{1} \iota_{2}+\imath_{1} \iota_{3}+l_{2} \iota_{3}\right) /\left(\left(l_{1} \iota_{2}+l_{2}+l_{1}\right)\left(l_{2}+l_{1}\right)\right),
\end{aligned}
$$

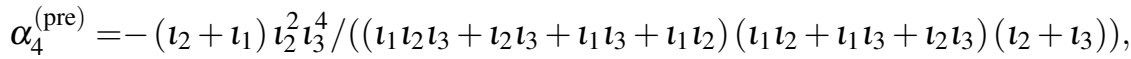

with $\imath_{1}=k_{n} / k_{n-1}, \iota_{2}=k_{n} / k_{n-2}, \iota_{3}=k_{n} / k_{n-3}$, as well as

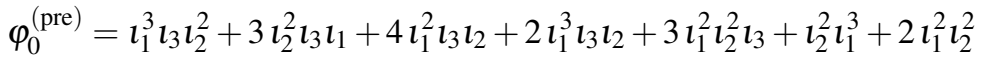

$$
\begin{aligned}
& +\imath_{2}^{2} \iota_{3}+2 \imath_{1} \imath_{2} \iota_{3}+\imath_{1} \imath_{2}^{2}+\imath_{3} \imath_{1}^{2}+\imath_{1}^{3} \iota_{3}+\imath_{1}^{3} \iota_{2}+\iota_{2} \imath_{1}^{2} \text {. }
\end{aligned}
$$

The predictor scheme (5) is implicit. However, since $M_{h}$ does not depend on $\tau$, it has to be factorised only once at the beginning and the factorisation can then be re-used in every time step. Hence, the predictor scheme is still computationally cheap.

The local truncation error of the predictor scheme is given by

$$
U^{(h)}\left(\tau_{n}\right)-\tilde{U}_{n}^{(h)}=C_{P}^{\mathrm{loc}} k_{n}^{5} \frac{\partial^{5} u}{\partial \tau^{5}}+\mathscr{O}\left(k_{n}^{6}\right)
$$

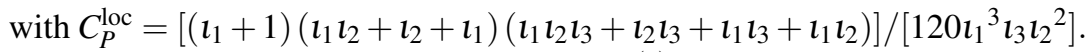

In the following, we use the notation $\tilde{U}_{n}^{(h)}$ to clarify whenever the predictor scheme is used to obtain the approximation of the solution $U^{(h)}\left(\tau_{n}\right)$.

Corrector scheme. For the corrector step, we use the implicit BDF-4 method with variable step-sizes to approximate the system of ordinary differential equations (4),

$$
\left[\alpha_{0}^{(\mathrm{cor})} M_{h}+k_{n} K_{h}\right] U_{n}^{(h)}=-M_{h} \sum_{j=1}^{4} \alpha_{j}^{(\mathrm{cor})} U_{n-j}^{(h)}+k_{n} g_{n}^{(h)}
$$

where 


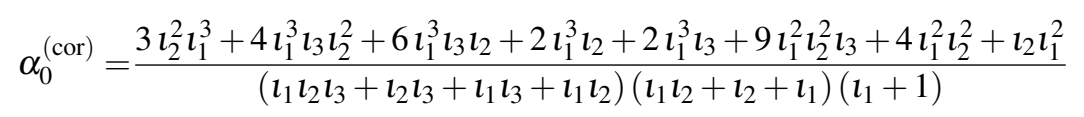

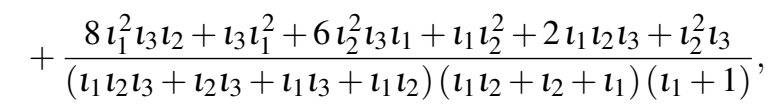

$$
\begin{aligned}
& \alpha_{1}^{\text {(cor) }}=-\frac{3 l_{2}^{2} l_{3} l_{1}+4 l_{1}^{2} l_{3} l_{2}+2 l_{1}^{3} l_{3} l_{2}+3 l_{1}^{2} l_{2}^{2} l_{3}+l_{1}^{3} l_{3} l_{2}^{2}+l_{2}^{2} l_{1}^{3}+2 l_{1}^{2} l_{2}^{2}+l_{2}^{2} l_{3}}{\left(l_{1} l_{2}+l_{1} l_{3}+l_{2} l_{3}\right)\left(l_{2}+l_{1}\right)} \\
& -\frac{2 \imath_{1} l_{2} l_{3}+l_{1} \imath_{2}^{2}+l_{3} \imath_{1}^{2}+l_{1}^{3} l_{3}+\imath_{1}^{3} l_{2}+l_{2} l_{1}^{2}}{\left(l_{1} l_{2}+l_{1} l_{3}+l_{2} l_{3}\right)\left(l_{2}+l_{1}\right)} \\
& \alpha_{2}^{\text {(cor) }}=\frac{\imath_{1}^{2} l_{2}^{2}+l_{1}^{2} \imath_{2}^{2} l_{3}+2 l_{1}^{2} l_{3} l_{2}+l_{2} l_{1}^{2}+l_{3} l_{1}^{2}+2 l_{2}^{2} l_{3} l_{1}+l_{1} l_{2}^{2}+2 l_{1} l_{2} l_{3}+l_{2}^{2} l_{3}}{\left(l_{1}+1\right)\left(l_{2}+l_{3}\right)}
\end{aligned}
$$

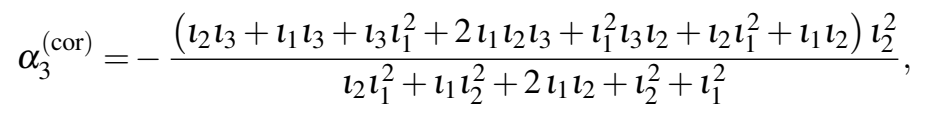

$$
\begin{aligned}
& \alpha_{4}^{\text {(cor) }}=\left(\imath_{2}+\imath_{1}+l_{1}^{2}+2 \imath_{1} \imath_{2}+\imath_{2} \imath_{1}^{2}\right) \imath_{2}^{2} \iota_{3}^{4} / \varphi_{4}^{\text {(cor) }}, \\
& \varphi_{4}^{\text {(cor) }}=\imath_{3}^{3} \imath_{1}^{2}+2 \imath_{1} \imath_{3}^{3} \imath_{2}+4 \imath_{1} \imath_{2}^{2} \imath_{3}^{2}+\imath_{2}^{2} l_{3}^{3}+2 \imath_{1}^{2} \imath_{2}^{2} \imath_{3}^{2}+\imath_{2}^{2} l_{3}^{3} \iota_{1}+\imath_{2}^{3} l_{3}^{2}
\end{aligned}
$$

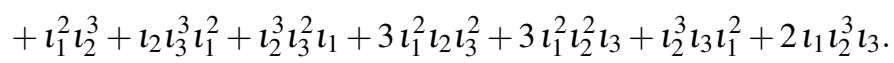

The local truncation error of the corrector scheme is given by

$$
U^{(h)}\left(\tau_{n}\right)-U_{n}^{(h)}=C_{C}^{\mathrm{loc}} k_{n}^{5} \frac{\partial^{5} U^{(h)}\left(\tau_{n}\right)}{\partial \tau^{5}}+\mathscr{O}\left(k_{n}^{6}\right)
$$

with

$$
\begin{aligned}
& C_{C}^{\mathrm{loc}}=-\left(l_{1} l_{2} l_{3}+l_{2} l_{3}+l_{1} l_{3}+l_{1} \imath_{2}\right)^{2}\left(l_{1}+1\right)^{2}\left(l_{1} l_{2}+l_{2}+l_{1}\right)^{2} / N_{C}^{\mathrm{loc}}, \\
& N_{C}^{\mathrm{loc}}=120 \imath_{1}^{3} \imath_{2}^{2} \iota_{3}\left[4 \imath_{1}^{3} \imath_{3} \imath_{2}^{2}+6 \imath_{3} \imath_{1}^{3} \imath_{2}+2 \imath_{3} \imath_{1}^{3}+3 \imath_{2}^{2} \imath_{1}^{3}+2 \imath_{2} \imath_{1}^{3}+8 \imath_{1}^{2} \imath_{3} \imath_{2}\right. \\
& \left.+9 l_{1}^{2} \imath_{2}^{2} \imath_{3}+l_{1}^{2} l_{3}+4 \imath_{1}^{2} \imath_{2}^{2}+\imath_{2} \imath_{1}^{2}+6 \imath_{2}^{2} l_{3} l_{1}+2 \imath_{1} l_{2} l_{3}+l_{1} l_{2}^{2}+l_{3} l_{2}^{2}\right] .
\end{aligned}
$$

Time-step adaption. The aim of the time-step adaption is to choose the time-step in such a way that the resulting local time-discretisation error stays below a given threshold $\hat{\varepsilon}>0$. Similar as in [PvS07], we use the local time-discretisation errors (6) and (8) to obtain the first order approximation

$$
\frac{\partial^{5} U^{(h)}\left(\tau_{n}\right)}{\partial \tau^{5}}=\frac{U_{n}^{h}-\tilde{U}_{n}^{h}}{k_{n}^{5}\left(C_{C}^{\mathrm{loc}}-C_{P}^{\mathrm{loc}}\right)}+\mathscr{O}\left(k_{n}\right) .
$$

The leading error term of the discretisation (7) can thus be approximated by

$$
\varepsilon_{n}=-\alpha_{0}^{(\mathrm{cor})} M_{h} C_{C}^{(\mathrm{loc})} k_{n}^{4} \frac{\partial^{5} U^{(h)}}{\partial \tau^{5}}=-\alpha_{0}^{(\mathrm{cor})} M_{h} C_{C}^{(\mathrm{loc})} \frac{U_{n}^{h}-\tilde{U}_{n}^{h}}{k_{n}\left(C_{C}^{\mathrm{loc}}-C_{P}^{\mathrm{loc}}\right)}
$$


The goal is now to choose the next step-size in time in a way that the norm of this error is bounded by the error threshold $\hat{\varepsilon}>0$ in a given norm. The general error structure is given by $\varepsilon_{n}=k_{n}^{4} \zeta\left(\tau_{n}\right) \Longleftrightarrow k_{n}=\left(\varepsilon_{n} / \zeta\left(\tau_{n}\right)\right)^{\frac{1}{4}}$ and thus we can, with $\left\|\varepsilon_{n}\right\| \leq \hat{\varepsilon}$, use $k_{n+1} \leq k_{n}\left(\hat{\varepsilon} /\left\|\varepsilon_{n}\right\|\right)^{\frac{1}{4}}$ to choose the new step size in time.

The approximation of the local discretisation error in time (10) can be nonsmooth, giving rise to abrupt changes of the chosen step size. To ensure that we avoid choosing a very large step size in case that the estimated error is very small, we introduce a small parameter $\beta>0$ (see [PvS07]) and adapt the time step size according to

$$
k_{n+1}=\left(\frac{\hat{\varepsilon}}{\hat{\varepsilon} \beta+\left\|\varepsilon_{n}\right\|}\right)^{\frac{1}{4}} k_{n}=: \xi_{n} k_{n}
$$

\section{Numerical results}

We consider the pricing of European Put options with model (1) and use $(S, v) \in$ $(1.5,600) \times(0.1,0.5)$. The computational domain is determined through the transformations given in Section 2. We choose step-size $h=\left(x_{\max }-x_{\min }\right) /(N-1)$ with $N=201$ steps in $x$-direction, in $y$-direction we begin at $y_{\min }$ and use step-size $h$. In (11), we set $\beta=0.01$. We use $K=100, T=2, r=0.05, \sigma=0.3, \kappa=1.1, \theta=0.3$, $\rho=-0.4$. For the start-up values, we apply the Crank-Nicolson time-steps with a fixed parabolic mesh ratio, choosing $k_{n}=0.05 h^{2}, n=1,2,3$.

Figure 1 shows the adaptation factor $\xi_{n}$, the positioning of the grid points in time, and the local error $\left\|\varepsilon_{n}\right\|_{2}$ for the GARCH model (left column) and the $a=b=3 / 4$ model (right column). For GARCH the algorithm leads to overall 104 grid-points in time. The local error remains just below the chosen threshold $\hat{\varepsilon}=0.001$, while time steps are increased. For GARCH, 50 of 104 grid-points in time, including the three initial points where Crank-Nicolson type time discretisation is used, are located in the interval $[0,0.01]$, i.e. $48 \%$ of the grid-points are positioned in only $0.5 \%$ of the time-domain. On the other hand only six points are placed in the time interval $[1,2]$. The results for the $a=b=3 / 4$ model show a similar behaviour. For comparison we repeat both simulations, now with the same numbers of equidistant time steps. Initially, the local error is above the threshold and later far below, indicating the sub-optimality of the equidistant distribution of points in time.

\section{References}

[CJM10] P. Christoffersen, K. Jacobs, and K. Mimouni. Models for S\&P500 dynamics: Evidence from realized volatility, daily returns, and option prices. Review of Financial Studies, 23:3141-3189, 2010. 

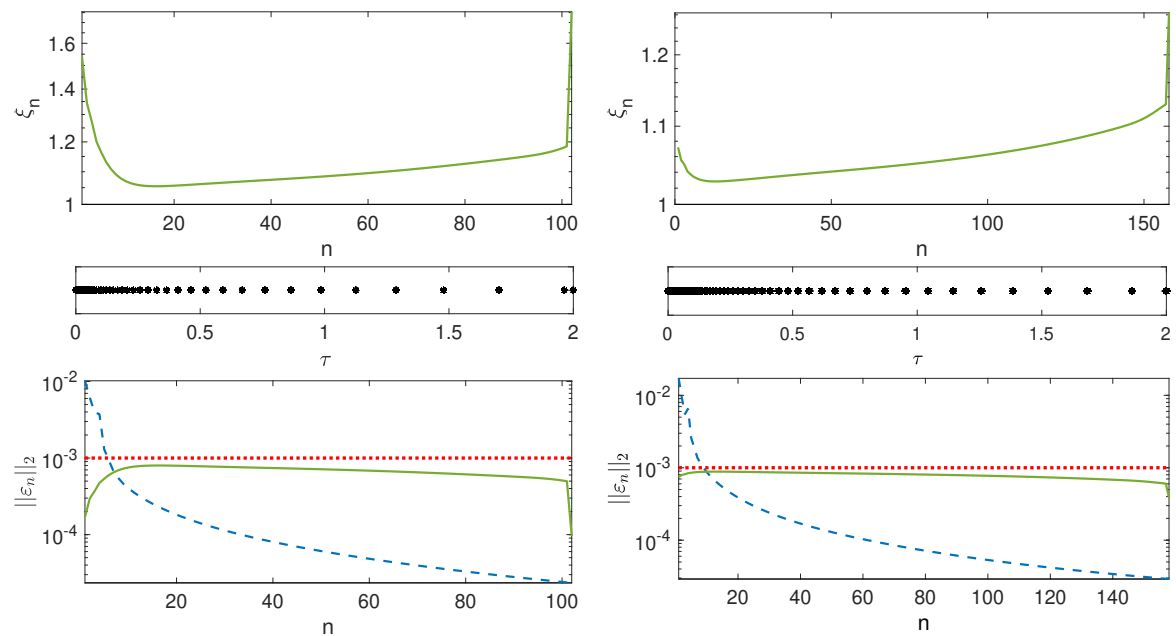

Fig. 1 Adaptation factor $\xi_{n}$, time grid points distribution, and error threshold $\hat{\varepsilon}$ (dotted red), local error $\left\|\varepsilon_{n}\right\|_{2}$ for adaptive (solid green) and equidistant time stepping (dashed blue): GARCH (left), $a=b=3 / 4$ model (right).

[DF12] B. Düring and M. Fournié. High-order compact finite difference scheme for option pricing in stochastic volatility models. J. Comput. Appl. Math., 236(17):4462-4473, 2012.

[DH15] B. Düring and C. Heuer. High-order compact schemes for parabolic problems with mixed derivatives in multiple space dimensions. SIAM J. Numer. Anal., 53(5):21132134, 2015.

[Dua95] J. Duan. The GARCH option pricing model. Math.Finance, 5(1):13-32, 1995.

[Hes93] S.L. Heston. A closed-form solution for options with stochastic volatility with applicatins to bond and currency options. Rev. Fin. Studies, 6(2):327-343, 1993.

[KTW70] H.O. Kreiss, V. Thomee, and O. Widlund. Smoothing of initial data and rates of convergence for parabolic difference equations. Commun. Pure Appl. Math., 23:241$259,1970$.

[Lew00] A.L. Lewis. Option valuation under stochastic volatility. Finance Press, Newport Beach, CA, 2000.

[LSRHF02] P. Lötstedt, S. Söderberg, A. Ramage, and L. Hemmingsson-Frändén. Implicit solution of hyperbolic equations with space-time adaptivity. BIT, 42(1):134-158, 2002.

[PvS07] J. Persson and L. von Sydow. Pricing European multi-asset options using a space-time adaptive FD-method. Computing and Visualization in Science, 10:173-183, 2007. 